\title{
Differenzierung und Professionalisierung der personenbezogenen Beratungsformate
}

\author{
Heidi Möller • Beate Fietze • Thomas Webers
}

Online publiziert: 22. April 2021

(C) Der/die Autor(en) 2021

Die Diskussion über den Stand der Professionalisierung und sowie über den Professionsanspruch personenbezogener Prozessberatung begleitet die fachliche Entwicklung der Beratung von ihren Anfängen an. Dabei hat sich diese Beratungsform inzwischen so erfolgreich etabliert, dass auch klassische Unternehmensberatungsfirmen, die ihre Dienstleistung vormals ausschließlich auf der Basis betriebswirtschaftlicher und juristischer Expertise anboten und großen Wert auf Distanz zu psychologisch orientierten Ansätzen legten, längst selbst Coaching, Teamentwicklung und Organisationsentwicklungsmaßnahmen anbieten. Diese steigende Akzeptanz geht auf der einen Seite mit immer weiteren Ausdifferenzierungen von Beratungsangeboten einher, die eine Orientierung im Feld der Beratung weiter erschweren. Auf der anderen Seite haben speziell die Beratungsangebote Supervision, Coaching und Organisationsentwicklung bereits eine so lange Erfahrung und Tradition, dass sich hier zunehmend konsistente Berufsbilder herauskristallisieren, ohne dass allerdings dieser Prozess als abgeschlossen betrachtet werden könnte. Noch immer ist „,Der Weg zum passenden Türschild“ (Reyer 2016, S. 472) nicht klar beschrieben. Keines der genannten Beratungsformate hat bisher einen formalen Professionsstatus, wie ihn zum Beispiel Ärzte oder Juristen für sich reklamieren können.

\footnotetext{
Prof. Dr. H. Möller $(\bowtie)$

Institut für Psychologie, Universität Kassel, Holländische Straße 36-38, 34127 Kassel, Deutschland E-Mail: heidi.moeller@uni-kassel.de

Dr. B. Fietze

artop GmbH. Institut an der Humboldt-Universität zu Berlin, Christburger Str. 4, 10405 Berlin, Deutschland

E-Mail: fietze@artop.de

T. Webers

Paulstraße 9, 53111 Bonn, Deutschland

E-Mail: tw@thomas-webers.de
} 
Im Verlauf der Institutionalisierungsgeschichte der personenbezogenen Prozessberatung scheinen inzwischen immerhin zwei grundlegende Perspektiven geklärt zu sein: So hat sich erstens inzwischen die kategoriale Unterscheidung zwischen der Prozessberatung und der Expertenfachberatung etabliert (Schein 2010), die auch dann erhalten bleibt, wenn beide Aspekte in einem Beratungsgeschehen zusammenfließen. Über eine reine Fachberatung hinausgehend macht die Prozessberatung den Prozess selbst zum Thema und beansprucht die Prozessberatung eine Professionalisierung des Beratungsgeschehens selbst. Zweitens konnte durch die Unterscheidung von Methode und Format bzw. Beratungsformen Klarheit über den Ansatzpunkt der Professionsbildung gewonnen werden (Buer 2007). Nicht die Methoden, sondern die Formate bilden die Basis für die Definition einer Profession, in deren Rahmen unterschiedliche wissenschaftsbasierte, theoretische Ansätze und methodische Vorgehensweise zur Anwendung kommen. In diesem Heft knüpfen wir an diese Überlegung an: Im Fokus steht die Frage nach dem Selbstverständnis und dem jeweiligen Zuständigkeitsanspruch, die mit den Beratungsangeboten verbunden werden. Es ist nämlich keineswegs klar, wie spezifisch die Problemlagen definiert werden können und wie spezifisch die Antworten der jeweiligen Beratungsformate ausfallen.

In ihrem Beitrag unterstreicht Kornelia Rappe-Giesecke den Professionsanspruch der berufs- und personenbezogenen Beratung im Sinne der Sonderstellung der Professionen - wohl wissend, dass noch viel konzeptionelle Klärungsarbeit geleistet werden muss. Denn bisher konnte sich noch keine Typologie von Beratungsformaten durchsetzen. Vor dem Hintergrund der „Triadischen Theorie der Praxis“ analysiert die Autorin das Kräftefeld der verschiedenen Praxissysteme, die an der sozialen Konstruktion der Zuständigkeit berufs- und personenbezogener Beratung beteiligt sind. Darauf aufbauend unterbreitet sie einen eigenen Vorschlag für eine Klassifikation und Typologie der Beratungsformen. Ansatzpunkt der Profession bildet dabei das Themenfeld der berufsbezogenen Beratung insgesamt, während die unterschiedlichen Beratungsformate als Beratungstypen fungieren. Aufgabe der Profession sieht die Autorin darin, sich auf deren Abgrenzung zu einigen und zur Fragestellung des Klienten in Beziehung zu setzen. Im Zuge der Auftragsklärung, der Vorphase des eigentlichen Beratungsgeschehens, kann dann die Berater/in die adäquate Passung der Problemlage und die Zuständigkeit der verschiedenen Beratungsformen wie Supervision, Coaching oder Organisationsentwicklung ermitteln und zur Anwendung bringen.

An dieser Stelle berühren sich die Fragen der fachlichen Typologie und Klassifikation mit dem konkurrierenden Autonomieanspruch und die soziale und kognitive Identität der Vertreter der verschiedenen Beratungsformate, wie sie sich im Laufe der Geschichte bereits herausgebildet haben. So haben Supervision und Coaching bekanntermaßen unterschiedliche historische Entstehungshintergründe, weisen aber gleichzeitig eine enge fachliche Nachbarschaft auf. Es stellt sich somit hier die Frage, ob die Unterscheidung dieser beiden Beratungsformate vorrangig aus ihrer Praxiserfahrung in unterschiedlichen sozialen Milieus und organisationalen Bezügen (Wirtschaftsunternehmen versus soziale Einrichtung) herrührt und inzwischen in einen identischen Zuständigkeitsbereich und ein identisches Kompetenzprofil gemündet sind oder ob auch heute weiterhin fachliche Unterschiede bestehen, aus denen sich unterschiedliche Zuständigkeitsbereiche ableiten lassen. 
Stefan Busse konstatiert, dass die Supervision schon lange keine (reine) Sozialarbeitsmethode mehr ist, sondern inzwischen als ein generalisiertes berufs- und arbeitsweltorientiertes Beratungsformat verstanden wird. Er rekonstruiert diese Entwicklungslinien als eine Gegenstands-, Zuständigkeits- und Kompetenzerweiterung. Dabei gerät vor allem die Entdeckung der Organisation durch die Supervision in den Fokus. Der Blick musste sich weiten, vom Individuum auf den Kontext. Insbesondere galt es, sich an der Nachbardisziplin Organisationsberatung zu reiben. Es folgte die Auseinandersetzung mit dem Coaching. All dies hat Klärungsprozesse befördert. Als zentrale Professionalisierungsanforderung für Supervision benennt der Autor den Umgang mit zentralen Widersprüchen der Spätmoderne.

Über die wichtigen historischen Stationen rekonstruieren Ingo Steinke und Johanna M. Steinke die Ausdifferenzierung und Professionalisierung von Coaching. Ausgehend von dem Einsatz von Coaches an den amerikanischen Universitäten zur Prüfungsvorbereitung und im Sport zeigen der Autor/innen, wie sich durch die veränderte Interpretation der Erfolgsfaktoren für den Coachee auch die Rollenanforderungen an den Coach/Trainer verändern. Zunehmend werden verhaltenspsychologische Ansätze aufgegriffen, und Coaching findet in der Sportpsychologie seine erste professionelle Verortung. Auch bei der Übertragung der Rolle des Coachs ins Management dominierte lange Zeit die unmittelbare leistungs- und ergebnisorientierte Unterstützung durch einen Coach. Erst durch die Integration der reflexiven Beratung aus der psychologischen Beratung und Therapie in das vormalige Coachingverständnis vollzieht Coaching die qualitative Wende und entwickelt sich zu einem neuen und eigenständigen Berufsbild.

Eine wichtige Rolle im Prozess der Professionalisierung nehmen auch die Fachund Berufsverbände ein. Sie sind die „Heimatsysteme“ für die Professionellen, in denen die Ergebnisse der fachlichen Themen in eine kollegiale Verbindlichkeit überführt werden. Bezogen auf diesen Kontext berichten Beate Fietze und Lutz Salamon von der Entwicklung des Roundtable Coaching e.V. (RTC) von seinen Anfängen im Jahr 2005 bis zur Gründung des Dachverbandes im Jahr 2020 und der Neufiguration der Verbändelandschaft. Von Beginn an war die Entwicklungsdynamik des RTC von der Spannung zwischen Konkurrenz und Kooperationsbereitschaft der Verbände geprägt. Nur langsam hat sich die wechselseitige Fremdbeobachtung angesichts einer diffusen Marktlage in ein Selbstverständnis gewandelt, das die Arbeit im RTC als Beteiligung an der Entwicklung eines geteilten Coachingverständnisses und als Engagement für die gesellschaftliche Anerkennung der neuen Profession verstand. Als entscheidend für die Entwicklungsdynamik stellten sich dabei jene Situationen heraus, an denen durch eine starke öffentliche Sichtbarkeit des RTC eine verbandsübergreifende Verbindlichkeit eingegangen werden musste. An diesen Wendepunkten zeigte sich, ob die Verbände primär an den verbandseigenen Interessen i. S. der Marktlogik oder an der Konstituierung eines professionellen Kollegiums und an einem verbandsübergreifenden Berufsbild orientiert sind.

Jörg Middendorf und Michaela Ritter machen auf eine potenziell disruptive Veränderung im Coaching-Markt aufmerksam. Große Plattformunternehmen, mit zum Teil mehreren Millionen Euro Venture-Kapital ausgestattet, bedrohen den klassischen Einzelunternehmermarkt. Sie locken damit, dass sie den Personalentwicklern in den Unternehmen und den Coaches ungeliebte Arbeit abnehmen: das Marketing. 
Während die Verbandslandschaft im Coaching eher heterogen erscheint, drängen nun also Marktfremde in die Domäne Coaching. Dies wirft ein neues Licht auf die Professionalisierungsdebatte. Die Autoren beleuchten diese Veränderungen mit verschiedenen aktuellen Umfragen. Ihr Fazit: „Da es die Forschung und die Verbände in den letzten zwanzig Jahren nicht geschafft haben, allgemeingültige Coaching-Standards zu entwickeln und zu verankern, setzen heute die Digital Coaching Provider als neue Marktteilnehmende eigene Standards“.

Neben diesen Beiträgen zu unserem Themenschwerpunkt bringen wir in diesem Heft einige Beiträge zu weiteren Themen: Philipp Ziegler-Rehak stellt den Forschungsstand zum Dual Career-Coaching vor. Sind doch heutige Studienabsolvent/ innen stets der Auffassung, sie würden einander die gleichen beruflichen Chancen einräumen, spricht die Realität eine andere Sprache. Meist geht die Schere der Karrieremöglichkeiten zulasten der Frauen auf. Die Geburt der Kinder und Mobilitätszwänge bzw. -entscheidungen werden dafür verantwortlich gemacht (vgl. Rusconi und Solga 2011). Dual Career Couples (DCCs) brauchen einen triadisch konzeptualisierten Beratungsraum, um einen Rahmen zu schaffen, der faire Aushandlungsprozesse ermöglicht. Ziegler-Rehak unterstützt die Debatte mit einer vergleichenden Darstellung und Einordnung von zehn bereits vorliegenden Beratungsansätzen und zeigt Perspektiven auf, wie die Weiterentwicklung des Dual Career Coachings erfolgen könnte.

In ihrem Praxisbericht stellen Sebastian Kunert und Stephan Bedenk die komplexe Projektarchitektur einer mehrjährigen Organisationsentwicklung vor und greifen gleichzeitig den Faden der Professionalisierungsdiskussion auf. Ausgehend von der allgemeinen Anerkennung der systemischen Organisationsentwicklung als einer eigenständigen Beratungsform schließen die Autoren zunächst an den Professionalisierungsdiskurs an, der die Ausdifferenzierung neuer Praxisfelder reflektiert. Professionalisierung macht sich aus ihrer Sicht jedoch nicht nur an den Abgrenzungsbemühungen der verschiedenen Formate fest. Stattdessen wenden sie den Blick auf die Binnendifferenzierung und Integration der verschiedenen Interventionsformen innerhalb der personenbezogenen Prozessberatung. In der Projektarchitektur kommen eine Vielzahl unterschiedlicher Formate bzw. Settings zur Anwendung wie z. B. Großgruppenmoderation, Führungskräfteklausuren oder Einzelcoaching. Erst durch die Integration dieser Formate auf den verschiedenen Interventionsebenen der Projektarchitektur lässt sich die intendierte Wirkung realisieren. Durch diese Perspektivenänderung erfährt der Format-Begriff einen anderen Stellenwert. In der Metapher des Hausbaus betrachten die Autoren die Formate oder Settings als „Gewerke“ und stellen die Frage, ob sich hinter einem „Gewerk" auch immer gleich eine Profession verbirgt. Mit dieser Frage knüpfen sie implizit an die konzeptionellen Überlegungen von Rappe-Giesecke an, die ein übergeordnetes Konzept der berufs- und personenbezogenen Beratung als Bezugspunkt der Professionsbildung vorschlägt.

Christiane Münderlein hat in ihrer qualitativen Interviewstudie Gelingensfaktoren und Herausforderungen geteilter Führung erhoben. Das postheroische Management schreit förmlich nach Konzepten für Doppelspitzen, der Forschungsstand dazu ist dürftig und divers. In ihrer Arbeit stellt Münderlein die Potenziale geteilter Führung heraus. Neben den Chancen dieses Modells stellt sie Fragen zur Passung und 
Komplementarität des „Paares“. Die Rolle des begleitenden Coachings bei unterschiedlichen Doppelspitzenmodellen wird anschaulich dargelegt, und die Autorin bleibt die Antwort des Beitrages von Doppelspitzen in der aktuellen Führungsdebatte nicht schuldig.

In der Rubrik Diskurs erwarten Sie zwei Beiträge, die sich den unser aller Alltag dominierenden pandemischen Geschehen zuwenden. „Zeitgemäßes über ein Virus und den Tod" überschreibt Stephan Schmidt seinen Beitrag. Er strengt einige klinische Beobachtungen an, wie die Pandemie sich in der therapeutischen Beziehung zeigt. Diese sind für Berater/innen im Organisationskontext sicher anregend und gut übertragbar. Insbesondere der Rückgriff auf die Arbeit Freuds in seiner Differenzierung zwischen Realangst und neurotischer Angst überzeugt. Hilfreich ist die Herleitung, inwiefern das gute innere Objekt vor der Todesangst schützen kann. Die Pandemie erinnert uns ständig an unsere Vergänglichkeit, die daher wieder mehr bewusst werden sollte.

Wolfgang Weigand reflektiert in fünfzehn Schritten unsere affektiven Reaktionen auf die Corona Pandemie. Ihm gelingt es, eine Systematik von individuellen und kollektiven Abwehrmechanismen aufzuzeigen, die hilft, nie Gefühltes, nie Gekanntes zu benennen. Anschaulich beschreibt er, welch ein hohes Maß an Spannungs- und Unsicherheitstoleranz wir als Gesamtgesellschaft, aber auch als Individuen aktuell aufbringen müssen. Er macht deutlich, dass wir uns in einem nie erlebten Maß mit Unsicherheit und Ohnmachtsgefühlen konfrontiert sehen. Mit Snowden und Boone (2007), den Begründern des Cynefin-Framework, ließe sich von einem chaotischen Systemzustand sprechen, wo keine Beziehung zwischen Ursache und Wirkung auszumachen ist. Hier ist der Ansatz ,handeln - beobachten - reagieren“ der politisch Verantwortlichen angemessen. Die Datenlage verändert sich beständig, Zielorientierung und Steuerung sind in chaotischen Systemen nicht möglich, auch wenn wir es uns noch so sehr wünschen. Verwirrung meint den Zustand des Nichtwissens darüber, welche Art von Kausalität besteht.

Funding Open Access funding enabled and organized by Projekt DEAL.

Open Access Dieser Artikel wird unter der Creative Commons Namensnennung 4.0 International Lizenz veröffentlicht, welche die Nutzung, Vervielfältigung, Bearbeitung, Verbreitung und Wiedergabe in jeglichem Medium und Format erlaubt, sofern Sie den/die ursprünglichen Autor(en) und die Quelle ordnungsgemäß nennen, einen Link zur Creative Commons Lizenz beifügen und angeben, ob Änderungen vorgenommen wurden.

Die in diesem Artikel enthaltenen Bilder und sonstiges Drittmaterial unterliegen ebenfalls der genannten Creative Commons Lizenz, sofern sich aus der Abbildungslegende nichts anderes ergibt. Sofern das betreffende Material nicht unter der genannten Creative Commons Lizenz steht und die betreffende Handlung nicht nach gesetzlichen Vorschriften erlaubt ist, ist für die oben aufgeführten Weiterverwendungen des Materials die Einwilligung des jeweiligen Rechteinhabers einzuholen.

Weitere Details zur Lizenz entnehmen Sie bitte der Lizenzinformation auf http://creativecommons.org/ licenses/by/4.0/deed.de. 


\section{Literatur}

Buer, F. (2007). Zehn Jahre Format und Verfahren in der Beziehungsarbeit. Zur Rezeption einer bedeutsamen Unterscheidung. Organisationsberatung, Supervision, Coaching, 14(3), 283-300.

Reyer, T. (2016). Beratungsdschungel? Beratung, Coaching, Therapie, Supervision und mehr Differenzierung von Arbeitsformaten der Prozessberatung. Organisationsberatung, Supervision, Coaching, 23(4), 463-467.

Rusconi, A., \& Solga, H. (Hrsg.). (2011). Gemeinsam Karriere machen. Die Verflechtung von Berufskarrieren und Familie in Akademikerpartnerschaften. Opladen: Barbara Budrich.

Schein, E. (2010). Prozessberatung für die Organisation der Zukunft. Der Aufbau einer helfenden Beziehung. Bergisch Gladbach: EHP.

Snowden, D., \& Boone, M.E. (2007). A leader's framework for decision making. Harvard Business Review, 85(11), 68-76. 